\title{
Optimal Duration of Antibiotics Therapy After Video- assisted Thoracoscopic Surgery in Thoracic Empyema and Complicated Parapneumonic Effusion
}

\section{Shungo Yukumi ( $\sim$ onsen2323@gmail.com )}

National Hospital Organization Ehime Medical Center Department of Surgery

Kei Ishimaru

Ehime University Medical School Department of Minimally Invasive Gastroenterology

Hideaki Suzuki

National Hospital Organization Ehime Medical Center Department of Surgery

Masamitsu Morimoto

National Hospital Organization Ehime Medical Center Department of Surgery

\section{Mayuko Senba}

National Hospital Organization Ehime Medical Center Respiratory medicine

\section{Tetsuya Yamamoto}

National Hospital Organization Ehime Medical Center Respiratory medicine

\section{Yoshifumi Sano}

Ehime University Medical School Department of Cardiovascular and Thoracic Surgery

\section{Masahiro Abe}

National Hospital Organization Ehime Medical Center Respiratory medicine

\section{Yu Mori}

Ehime University Medical School Department of Cardiovascular and Thoracic Surgery

\section{Nobuhiko Sakao}

Ehime University Medical School Department of Cardiovascular and Thoracic Surgery

\section{Research Article}

Keywords: Antibiotics Therapy, Thoracoscopic Surgery, Complicated Parapneumonic Effusion

Posted Date: October 28th, 2021

DOl: https://doi.org/10.21203/rs.3.rs-997272/v1

License: (c) (1) This work is licensed under a Creative Commons Attribution 4.0 International License. Read Full License 
Page 2/8 


\section{Abstract}

\section{Background}

There is no evidential report about the optimal duration of antibiotic use following video-assisted thoracoscopic surgery debridement (VATS-D) in thoracic empyema (TE) or complicated parapneumonic effusion (PPE). The purpose of this study was to determine the optimal duration of antibiotic therapy after VATS-D for TE /PPE.

\section{Methods}

Between January 2011 and December 2019, total thirty-three patients (28 men, 5 women; median age 63 years) corresponding to ACCP category 3 or 4 receiving VATS-D were included in the study. Time until the body temperature (BT) was to be less than $37.5^{\circ} \mathrm{C}$ and $37.0^{\circ} \mathrm{C}$, WBC count to be less than $10,000 / \mu$ land segmented neutrophil (seg) count to be less than $80 \%$ were retrospectively analyzed.

\section{Results}

The median time from the onset of TE/PPE to the operation was 13 days. Pre and postoperative antibiotic had median values of 5 and 7 days, respectively. There were no hospital deaths within 30 days of the operation. Major complications occurred in 4 cases (3 respiratory failures and one celebral infarction). Median postoperative hospital stay was 14 days. Success rate in TE/PPE treatment was $88 \%$. The median number of days until the conditions met were $\mathrm{BT}>37.5^{\circ} \mathrm{C}$ for 3 days, $\mathrm{BT}>37.0^{\circ} \mathrm{C}$ for 6 days, WBC $<10,000$ for 4 days and seg $<80 \%$ for 7 days.

\section{Conclusion}

The optimal antibiotic duration of antibiotic use after VATS-D for TE/PPE is approximately 5 days. Urgent VATS-D will shorten the total antibiotic duration.

\section{Introduction}

Antibiotics treatment for thoracic empyema (TE) or complicated parapneumonic effusion (PPE) is always empiric. Usually, antibiotic treatment starts when drainage or surgical intervention is planned. The target bacteria are often unknown when the antibiotics are chosen. Bacterial cultures tend to prove negative so antibiotic treatment often has to be continued empirically.

Recommendations regarding the choice of antibiotics are described in guidelines such as the Sanford Guide [1], British Thoracic Society (BTS) pleural disease guideline [2] or Japanese Association for Infectious Disease/Japanese Society of Chemotherapy (JAID/JSC) guideline. According to the patient's risk and community or hospital-acquired status, the regimens are suggested in these guidelines but there is no evidential description about the optimal duration of antibiotic therapy. 
Video-assisted thoracoscopic surgery debridement (VATS-D) is a common and evidential treatment for TE/complicated PPE [3]. It shortens drainage duration or hospital stay [4]. However, the appropriate duration of antibiotic therapy following surgical intervention is unclear. Although it is said from a few to several weeks of antibiotic administration is required, we thoracic surgeons may not have an image that such long-term antibiotic administration is required after VATS-D in acute TE or complicated PPE.

The purpose of this study was to determine the optimal duration of antimicrobial therapy after VATS-D in TE/PPE from the point of view of the physical findings and laboratory data.

\section{Material And Methods}

Data were retrospectively collected from the registry of Ehime Medical Center. The study protocol was approved by the Review Board of National Hospital Organization Ehime Medical Center, and the written informed consent was waived by the institutional Review Board of Ehime Medical Center because our study was retrospective in design. The study procedure was performed according to Declaration of Helsinki. Cases receiving VATS-D for TE/PPE at Ehime Medical Center in the period from 2010 to 2019 were identified. Patients who fulfilled Category 3 or 4 of the American College of Chest Physicians (ACCP) Categorizing Risk for Poor Outcome in Patients with PPE [5] after a thoracentesis, simple drainage or intraoperative collection of pleural effusion were identified and included in this study. Chronic empyema or empyema with bronchopleural fistula were excluded.

VATS-D procedures were performed under general anesthesia and one lung ventilation for all cases. Two or three trocars were placed for a thoracoscope or other instruments. The pleural cavity was debrided, loculated cavity was disrupted and fibrous adhesions were removed to make it into one cavity and reexpand the lung.

The following parameters were evaluated (1) preoperative characteristics; age, sex, comorbidities, white blood cell count (WBC), percentage of segmented neutrophils, serum C-reactive protein level (CRP), antibiotic administration status, time from the onset of the disease to VATS-D, and bacterial culture. (2) Postoperative characteristics; hospital stay, chest tube duration, major postoperative complications corresponding to Clavien-Dindo Classification grade $\mathbb{Q}$ or higher, rehospitalization due to recurrence within 1 year, antibiotic administration status, period until the body temperature confirmed to be less than 37.5 ${ }^{\circ} \mathrm{C}$ and $37.0^{\circ} \mathrm{C}, \mathrm{WBC}$ to be less than $10,000 / \mu \mathrm{l}$, and segmented neutrophil to be less than $80 \%$.

\section{Results}

In the period studied, 33 patients received VADS-D for TE/PPE (Table 1). Twenty-six cases (79\%) were ACCP category 3 and 7 cases were 4 (21\%). Thirty-one patients (91\%) had primary TE/PPE and $3(9 \%)$ had secondary TE/PPE. Among the primary cases, chest computed tomography demonstrated pneumonia in 25 cases and lung abscess in one case. They were considered to be the cause of TE/PPE. In rest 5 cases (15\%), the cause was unknown. Three secondary cases followed lobectomy for lung 
cancer, percutaneous transhepatic gallbladder drainage for acute cholecystitis and traumatic hemopneumothorax.

The time from the onset or the diagnosis of TE/PPE to VATS-D was approximately two weeks (Table 2). Six mental disorder cases ( 5 schizophrenia and 1 severe depression) were included in this study (Table 1). Although, it is difficult to determine the onset of TE/PPE in these patients, the day complaint of fever, chest pain or dyspnea was noticed by the patient or a caregiver was set as the onset. The preoperative period in these cases tended to be longer than in other cases with a mean of 25 vs 14 days and a median of 30 vs 13 days, respectively.

The median postoperative hospital stay was 14 days. Postoperative drainage duration was about a week. Major postoperative complications corresponding to Clavien-Dindo Classification grade $\otimes$ or higher occurred in 4 cases (Table 2). Postoperative acute respiratory failure requiring ventilator management was observed in 3 patients, and cerebral infarction was observed in one patient. No death in the hospital was observed within 30 days after surgery. Recurrence and progression to chronic empyema were seen in 3 cases and 1 case, respectively. The recurrent cases were treated by simple drainage and chronic empyema was treated by omentoplasty.

Bacterial culture from pleural effusion was positive in 12 cases (36\%). Streptococcus in 7 cases, anaerobic bacteria in 3 cases and staphylococcus, neisseria, prevotella in 1 case were identified. Fluoroquinolone resistance was observed in 2 cases of anaerobic bacteria, and CLDM resistance was observed in 1 case, but no problematic resistant bacteria were observed.

The preoperative and post-operative antibiotic choice are shown in Table 3. Carbapenem or tazobactam/piperacillin (TAZ/PIPC) was selected in more than $60 \%$ of cases as an initial agent. Of these cases, 8 had postoperative conversion into fluoroquinolone or cephalosporin. On the other hand, switching to carbapenem or TAZ/PIPC from another agent was performed in 5 cases. During the postoperated course, 3 patients had converted the antibiotics (from cephalosporin to fluoroquinolone or sulbactum/ampicillin). In four cases, oral administration of levofloxacin (LVFX) was added before discharge.

Postoperative inflammatory parameters required 7 days to return to the normal values (Table 4). Body temperature was measured at least 3 times per day. Laboratory examination was performed routinely on the day after surgery and added when deemed necessary. A mean of 5 and median 4 laboratory examinations were performed postoperatively before discharge.

\section{Discussion}

In TE/PPE therapy, the choice of antibiotics is always empiric. In the present study, broad spectrum agents such as carbapenem or broad spectrum penicillin were used in almost all cases as an initial antibiotics. The choice depends on the patient's risk status or whether the empyema is communityacquired or nosocomial. In selecting the initial antibiotics, the target bacteria are often unknown so 
patients are treated empirically without culture data. Microbiological diagnosis is said to be achieved in only over $50 \%$ of cases [6] and it takes approximately a week to recognize the microbial etiologies. This means that culture data often do not reflect the full disease process and that the treatment is not always based on the culture data. Only Gram staining from the pleural effusions and peripheral blood or properties of the pleural effusions are the tips of the target bacteria. Thus, choosing broad spectrum agents is unavoidable in the induction of TE/PPE treatment.

When switching the antibiotics from broad to narrow spectrum agents pre-and postoperatively the decision should be made empirically because the target often remains invisible. The reasons for switching to narrow agents in our study were the preoperative fever reduction or intraoperative findings showing only serous effusion in all loculated cavities. On the other hand, when converting the antibiotics into broad agents, the decision is often made based on prolonged fever elevation or the remaining of the high level of WBC count. These decisions are not based on the result of bacterial culture so it does not correspond to so-called de-escalation or escalation of antibiotics. The ACCP category of the pleural effusion was 3 in 26 cases (79\%). Preoperatively, pleural effusion was always obtained from one of the loculated cavities. Disrupting the loculated cavity in VATS-D, even in the case of preoperative diagnosis was category 3 , often proving that the contents of cavities were homogenous and that some cavity contains pus. Therefore, the differential diagnosis of categories 3 and 4 or PPE and TE is often unclear. Thus, PPE and TE were examined without distinction in this study.

This study may be deviate from the international standards because metronidazole (MNZ) is not used and procalcitonin is not measured as an inflammatory marker. This is attributed to the special circumstances in Japan, intravenous MNZ and measuring procalcitonin were not commercially available or not covered by health insurance until late 2014 and early 2016. Currently, Japanese JAID/JSC recommends MNZ in combination with ABPC or 4th generation cephalosporin as a second choice regimen in both multidrug resistance risk and risk-free cases. For the purpose of suppressing the use of carbapenem to avoid antimicrobial resistance, MNZ may be chosen much in the future. Serum procalcitonin level-guided antibiotic use is reported to reduce antibiotic use [6]. Serum level of procalcitonin rise or fall rapidly in bacterial infection with high sensitivity and specificity. It may be a useful biomarker for diseases such as TE/PPE that require empiric discontinuation or changes in antibiotics.

VATS-D is an evidential treatment for TE/PPE. It is said to shorten the chest tube duration and hospital stay [4] and reduce hospital death or one-year mortality compared with simple drainage or intrapleural fibrinolytic therapy $[6,8]$. The reported success rate in VATS-D is approximately $90 \%[3,6,8]$. However, descriptions about the duration of antibiotic use are few. In our study, the duration of antibiotic use was 2 weeks. Although the duration of antibiotics was shorter than the description in guidelines, the success rate in our study was $88 \%$ whichand it was similar to previous reports. On the other hand, it took two weeks from the onset to the operation. Urgent VATS-D is required to shorten the total hospital stay and the duration of antibiotic use. 
There is no evidential consensus on the optimal duration of antibiotic therapy after surgical intervention in TE/PPE. The Sanford Guide states 4-6 weeks [1]. In BTS guidelines, it is stated that approximately 3 weeks may be optimal [2]. These descriptions include both surgical and nonsurgical cases. There seems to be no consensus on when to quit antibiotic therapy, especially after surgery. In the present study, a median fever exceeding $37.5^{\circ} \mathrm{C}$ remained for 3 days. The WBC count and percentage of segmented neutrophils took from 4 to 7 days to return to the normal baseline value. Discontinuation of antibiotics was decided based on the patient's physical or laboratory data. The mean and median postoperative duration of antibiotic use in this study was 7 days. Preoperatively antibiotics were used approximately 5 days. Antibiotic treatment in their primary care doctors was not reflected in the data we used. However, since the time from the onset to surgery was approximately 2 weeks, the total preoperative antibiotics are expected to be up to 2 weeks at the longest. As a result, the total antibiotic duration, including post and preoperation can be estimated to be 3 weeks in this study, and the postoperative antibiotic duration following VATS-D must be approximately 5 days.

\section{Conclusions}

The parameters considered in the study suggested that the routine antibiotic duration following VATS-D for TE/PPE is approximately 5 days. Empiric use of broad spectrum antibiotics is unavoidable in many cases. Urgent VATS-D and close observation of the patient's physical or laboratory data must reduce the long-term administration of broad spectrum antibiotics.

\section{References}

1. Gilbert, D. N., Chambers, H. F., Saag, M. S. \& Pavia, A. T. THE SANFORD GUIDE To Antimicrobial Therapy 2020, 50th Edition. Antimicrobial Therapy, Inc. Virginia.

2. Davies, H. E., Davies, R. J. O. \& Davies, C. W. H. Management of pleural infection in adults: British Thoracic Society Pleural Disease Guidline 2010. Thorax 2010; 65 supple 2: ii41-53. doi: 10.1136/thx.2010.137000.

3. Luh, S. P., Chou, M. C., Wang, L. S., Chen, J. Y. \& Tsai, T. P. Video-assisted thoracoscopic surgery in the treatment of complicated parapneumonic effusions or empyemas: outcome of 234 patients., 127 (4), 1427-1432 https://doi.org/10.1378/chest.127.4.1427 (2005).

4. Wait, M. A., Sharama, S., Hohn, J. \& Dal Nogare, A. A randomized trial of empyema therapy., 111 (6), 1548-1551 https://doi.org/10.1378/chest.111.6.1548 (1997).

5. Koegelenberg, C. F., Diacon, A. H. \& Bolliger, C. T. Parapneumonic pleural effusion and empyema. Respiration, 75 (3), 241-250 https://doi.org/10.1159/000117172 (2008).

6. Godfrey, S. M., Bramley, T. K. \& Detterbeck, F. Medical and surgical management of empyema. Semin Respir Crit Care Med, 40 (3), 361-374 https://doi.org/10.1055/s-0039-1694699 (2019).

7. Soni, N. J. et al. Procarcitonin-guided antibiotic therapy: a systematic review and meta-analysis. $J$ Hosp Med, 8 (9), 530-540 https://doi.org/10.1002/jhm.2067 (2013). 
8. Nayak, R., Brogly, S. B., Lajkosz, K., Lougheed, M. D. \& Petsikas, D. Outcomes of oprerated and nonoperated treatment of thoracic empyema: A population-based study. Ann Thorac Surg, 108 (5), 1456-1463 https://doi.org/10.1016/j.athoracsur.2019.05.090 (2019).

\section{Tables}

Due to technical limitations, table 1, 2, 3 and 4 is only available as a download in the Supplemental Files section.

\section{Supplementary Files}

This is a list of supplementary files associated with this preprint. Click to download.

- TEPPETable122.xlsx 